were associated with a reduced risk of HCC (Figure 1), with adjusted hazard ratios (aHR) (95\% confidence interval [CI]) of $0.55(0.42,0.71), 0.52(0.41,0.64), 0.47(0.38,0.58)$ and 0.46 $(0.37,0.56)$, respectively (all $\mathrm{p}<0.001)$. In contrast, ALT 1 to 2 times the ULN and ALT greater than 2 times the ULN at 12 months were associated with a higher risk of HCC as compared to ALTN at 12 months, with aHR (95\% CI) of $2.07(1.67,2.56)$ and $3.21(2.32,4.44)$, respectively, after adjustment for baseline ALT and important covariates.

Conclusions Early on-treatment ALTN reduces the risk of HCC in CHB patients having entecavir/TDF treatment. Ontreatment ALT above 1 and 2 times the ULN at 12 months were associated with higher risk of HCC.

\section{IDDF2018-ABS-0067 EFFICACY AND SAFETY OF GLECAPREVIR/ PIBRENTASVIR IN PATIENTS WITH HCV GENOTYPE 5 OR 6 INFECTION: THE ENDURANCE-5, 6 STUDY}

\begin{abstract}
${ }^{1}$ Tarik Asselah*, ${ }^{2}$ Tram Tran, ${ }^{3}$ Katia Alves, ${ }^{4}$ Tuan Nguyen, ${ }^{5}$ Florence Wong, ${ }^{6}$ Adam Mahomed, ${ }^{7}$ Seng Gee Lim, ${ }^{8}$ Samuel S Lee, ${ }^{9}$ Armand Abergel, ${ }^{10}$ Joe Sasadeusz, ${ }^{3}$ Preethi Krishnan, ${ }^{3}$ Zhenzhen Zhang, ${ }^{3}$ Ariel Porcalla, ${ }^{3}$ Roger Trinh, ${ }^{11}$ Edward Gane. ${ }^{1}$ Centre de Recherche sur I'Inflammation, INSERM UMR 1149, Universite Paris Diderot, Department of Hepatology, AP-HP Hopital Beaujon, Clichy, France; ${ }^{2}$ Cedars Sinai Medical Center, Los Angeles, California, USA; ${ }^{3}$ AbbVie Inc., North Chicago, Illinois, USA; ${ }^{4}$ Research and Education, Inc., San Diego, California, USA; ${ }^{5}$ Toronto General Hospital, University of Toronto, Toronto, Ontario, Canada; ${ }^{6}$ Faculty of Health Sciences, University of Witwatersrand, Charlotte Maxeke Johannesburg Academic Hospital, Department of Gastroenterology and Hepatology, South Africa; 'Division of Gastroenterology and Hepatology, National University Health System, Singapore; ${ }^{8}$ University of Calgary, Calgary, $A B$, Canada; ${ }^{9} \mathrm{CHU}$ Estaing, Clermont Ferrand, France; ${ }^{10}$ Royal Melbourne Hospital, Melbourne, Australia; ${ }^{11}$ Auckland Clinical Studies, Auckland, New Zealand
\end{abstract}

\subsection{6/gutjnl-2018-IDDFbestabstracts. 17}

Background The pangenotypic direct-acting antivirals (DAAs) glecaprevir (developed by AbbVie and Enanta)/pibrentasvir (G/P) are approved to treat chronic HCV genotype (GT)1-6 infection. In Phase 2 and 3 studies, G/P achieved high SVR12 rates with no virologic failures in 80 patients with GT5 or 6 infection. To increase the body of data for these genotypes, ENDURANCE5,6 evaluates patients from countries where GT5 and GT6 are endemic, such as South Africa (GT5), Myanmar and Vietnam (GT6). This study evaluates the efficacy and safety of G/P in patients with chronic HCV GT5 or GT6 infection.

Methods ENDURANCE-5,6 is an ongoing phase $3 \mathrm{~b}$, non-randomised, open-label, multicenter study conducted in adults with chronic HCV GT 5 or 6 infections with or without compensated cirrhosis who are HCV treatment-naive or experienced with interferon (IFN) or pegIFN with or without ribavirin (RBV) or sofosbuvir and RBV with or without pegIFN. G/P $(300 \mathrm{mg} / 120 \mathrm{mg})$ was orally dosed once-daily for 8 or 12 weeks in patients without or with compensated cirrhosis, respectively. The primary efficacy endpoint was SVR12. Secondary endpoints were on-treatment virologic failure or relapse. Adverse events and clinical laboratory abnormalities were monitored in all patients.

Results Seventy patients have enrolled to date, 61 and 9 in the 8 - and 12 week treatment arms, respectively; 66 have completed treatment. Baseline demographics are shown in table 1. The SVR4 among patients with available data is 61/ 62 (98\%). One HCV GT6c-l-infected patient with compensated cirrhosis experienced virologic breakthrough at treatment week 12, and one HCV GT5a-infected patient without compensated cirrhosis who achieved SVR4 relapsed at post-treatment week 12 . To date, three patients $(4 \%)$ have experienced treatment-emergent serious adverse events, none of which were related to $\mathrm{G} / \mathrm{P}$ or led to discontinuation; no Grade 3 alanine aminotransferase elevations have occurred.

\begin{tabular}{lll}
$\begin{array}{l}\text { Abstract IDDF2018-ABS-0067 Table 1 } \\
\text { and Disease Characteristics }\end{array}$ & Baseline Demographics \\
\hline Characteristics & 8 week G/P No Cirrhosis & 12 week G/P Cirrhosis \\
& $\mathrm{n}=61$ & $\mathrm{n}=9$ \\
\hline Female, n (\%) & $36(59)$ & $4(44)$ \\
Asian, n (\%) & $38(62)$ & $5(56)$ \\
Age, median years (range) & $57(24-79)$ & $65(32-75)$ \\
Genotype 5, n (\%) & $20(33)$ & $3(33)$ \\
Genotype 6, n (\%) & $41(67)$ & $6(67)$ \\
GT6a/6b & $19(31)$ & $1(11)$ \\
GT6c-I & $22(36)$ & $5(56)$ \\
HCV treatment-naive, n (\%) & $55(90)$ & $8(89)$ \\
Baseline HCV RNA, median $\log _{10}$ IU/ & $6.85(4.6-7.5)$ & $6.29(4.9-7.2)$ \\
ML (range) & & \\
\hline
\end{tabular}

Conclusions In this ongoing dedicated study, HCV genotype 5- and 6-infected patients without and with compensated cirrhosis treated with G/P for 8 and 12 weeks, respectively, achieved high rates of SVR4. Complete SVR4 and available SVR12 data will be presented.

\section{IDDF2018-ABS-0082 QUANTIFICATION OF CIRCULATING MIR- 125B-5P PREDICTS SURVIVAL IN CHRONIC HEPATITIS B PATIENTS WITH ACUTE-ON- CHRONIC LIVER FAILURE}

Enqiang Chen*, Yachao Tao, Juan Liao, Duoduo Lv, Yonghong Wang, Dongbo Wu, Dongmei Zhang, Hong Tang, Menglan Wang. West China Hospital of Sichuan University, China

\subsection{6/gutjnl-2018-IDDFbestabstracts. 18}

Background This study aimed to analyse serum levels of miR$125 \mathrm{~b}-5 \mathrm{p}$ in CHB patients with varying degrees of liver damage and evaluate its value in predicting the short-term outcome of acute-on-chronic liver failure (ACLF).

Methods CHB patients with normal hepatic function $(n=100)$, moderate-to-severe liver damage $(n=90)$ and $\operatorname{ACLF}(n=86)$ were included. Serum levels of miR-125b-5p and miR-122 were measured by quantitative real-time PCR.

Results Serum miR-125b-5p levels were increased along with disease progression, and lower in survival than in death among HBV-ACLF patients. Among HBV-ACLF patients, serum miR125b-5p were positively correlated with total bilirubin(TBil) $(\mathrm{r}=0.214, \mathrm{p}<0.05)$ and MELD score $(\mathrm{r}=0.382, \mathrm{p}<0.01)$, and negatively correlated with prothrombin activity $(\mathrm{PTA})(r=-0.215$, $\mathrm{p}<0.05)$. Interesting, serum miR-122 showed a completely opposite performance as compared to that of serum miR-125b5p. Cox Regression Analysis showed that serum miR-125b-5p, miR122 and PTA were all independent survival predictors of HBV-ACLF; and low miR125b-5p and high miR122 may predict 
longer survival of HBV-ACLF. Additionally, ROC analysis showed that miR125b-5p(AUC $=0.814)$ had higher performance for survival prediction of HBV-ACLF as compared to miR-122 $(\mathrm{AUC}=0.804), \quad \mathrm{PTA}(\mathrm{AUC}=0.762) \quad$ MELD score $(\mathrm{AUC}=0.799)$ and TBil(AUC $=0.670$ ) alone; and predictive effectiveness of miR $125 \mathrm{~b}-5 \mathrm{p}$ was greatly increased by the combination of $\operatorname{miR} 122$ (AUC $=0.898$ ).

Conclusions The increasing of serum miR-125b-5p is associated severity of liver damage, and high serum miR-125b-5p may serve as a novel predictor of poor outcome of HBVACLF.

\section{IDDF2018-ABS-0106 TENOFOVIR ALAFENAMIDE (TAF) COMPARED WITH TENOFOVIR DISOPROXIL FUMARATE (TDF) IN PATIENTS WITH CHRONIC HBV: WEEK 96 EFFICACY AND SAFETY RESULTS IN CHINESE PATIENTS ENROLLED IN 2 GLOBAL PHASE 3 STUDIES}

${ }^{1}$ Henry Lik-Yuen Chan*, ${ }^{2}$ Wai Kay Seto, ${ }^{3}$ Scott Fung, ${ }^{4}$ Jinlin Hou, ${ }^{5}$ Calvin Pan, ${ }^{6}$ WanLong Chuang, ${ }^{7}$ Ting-Tsung Chang, ${ }^{8}$ Aric-Josun Hui ${ }^{8}$, Chi-Yi Chen, ${ }^{10}$ Tak Yin Owen Tsang, ${ }^{11}$ Jenny Yang, ${ }^{11}$ John Flaherty, ${ }^{11}$ Anuj Gaggar, ${ }^{11}$ Shuyuan Mo, ${ }^{12}$ Jia-Horng Kao. ${ }^{1}$ Department of Medicine and Therapeutics, Institute of Digestive Disease and State Key Laboratory of Digestive Disease, The Chinese University of Hong Kong, Hong Kong; ${ }^{2}$ Queen Mary hospital, Hong Kong; ${ }^{3}$ Toronto General Hospital, Toronto, ON, Canada; ${ }^{4}$ Nanfang Hospital, Guangzhou, China; ${ }^{5}$ New Discovery LLC, Flushing, NY, United States; ${ }^{6}$ Kaohsiung Medical University Hospital, Kaohsiung, Taiwan; 'National Cheng Kung University Hospital, Tainan, Taiwan; ${ }^{8}$ Alice Ho Miu Ling Nethersole Hospital, Hong Kong; ${ }^{9}$ Chiayi Christian Hospital, Chiayi, Taiwan; ${ }^{10}$ Princess Margaret Hospital, Hong Kong; ${ }^{11}$ Gilead Sciences, Foster City, CA, United States; ${ }^{12}$ National Taiwan University Hospital, Taipei, Taiwan

\subsection{6/gutjnl-2018-IDDFbestabstracts.19}

Background TAF, a novel prodrug of tenofovir, has demonstrated efficacy noninferior to that of TDF at Week 48 in patients with chronic HBV (CHB) with significantly reduced bone and renal effects. Here we evaluated the efficacy and safety of TAF vs TDF in the subset of patients of Chinese ethnicity enrolled in two overseas studies.

Methods In 2 Phase 3 studies, HBeAg-negative (Study 108) and HBeAg-positive (Study 110) CHB patients were randomised 2:1 to TAF $25 \mathrm{mg}$ QD or TDF $300 \mathrm{mg}$ QD and treated for 96 weeks. In this analysis, the efficacy (HBV DNA).

Results Of 1298 patients randomised and treated, 471 (36\%) were of Chinese ethnicity; 156 (TAF 97; TDF 59) and 315 (TAF 207; TDF 108) patients were HBeAg-negative and HBeAg-positive, respectively. For the TAF and TDF groups within each study, baseline characteristics of the study populations were generally similar. Key efficacy results at Weeks 48 and 96 are presented in the Table (table 1). In both HBeAgnegative and $\mathrm{HBeAg}$-positive patients of Chinese ethnicity, the antiviral efficacy of TAF was similar to that of TDF and results were comparable to those in the overall overseas population. Numerically higher percentages of patients treated with TAF achieved normalisation of serum ALT values and antiHBe seroconversion at Weeks 48 and 96. The safety of TAF and TDF, including changes in renal and bone parameters, were similar to results previously reported in the overall overseas population.

Conclusions In CHB patients of Chinese ethnicity, TAF $25 \mathrm{mg}$ showed similar antiviral efficacy to TDF $300 \mathrm{mg}$, with less change in bone and renal parameters. Results in this subgroup were comparable to those in the overall population.
Abstract IDDF2018-ABS-0106 Table 1 Efficacy Results at Week 48 and 96

\begin{tabular}{|c|c|c|c|c|}
\hline \multirow[b]{2}{*}{$\mathrm{n} / \mathrm{N}(\%)$} & \multicolumn{2}{|c|}{ HBeAg-negative } & \multicolumn{2}{|c|}{$\begin{array}{l}\text { HBeAg-positive Patients } \\
\text { (Study 110) }\end{array}$} \\
\hline & $\begin{array}{l}\text { TAF }(n= \\
97)\end{array}$ & $\begin{array}{l}\text { TDF }(n= \\
59)\end{array}$ & $\begin{array}{l}\text { TAF }(n= \\
\text { 207) }\end{array}$ & $\begin{array}{l}\text { TDF }(n= \\
108)\end{array}$ \\
\hline \multicolumn{5}{|l|}{ Week 48} \\
\hline HBV DNA $<29 \mathrm{IU} / \mathrm{mL}$ & $90(93 \%)$ & $55(93 \%)$ & $143(69 \%)$ & $76(70 \%)$ \\
\hline $\begin{array}{l}\text { ALT normalisation (AASLD } \\
\text { criteria) }^{\mathrm{a}}\end{array}$ & $\begin{array}{l}46 / 94 \\
(49 \%)\end{array}$ & $\begin{array}{l}18 / 59 \\
(31 \%)\end{array}$ & $\begin{array}{l}100 / 207 \\
(48 \%)\end{array}$ & $\begin{array}{l}39 / 108 \\
(36 \%)\end{array}$ \\
\hline HBeAg loss & $N A^{b}$ & NA & $\begin{array}{l}28 / 204 \\
(14 \%)\end{array}$ & $\begin{array}{l}11 / 103 \\
(11 \%)\end{array}$ \\
\hline HBeAg seroconversion & NA & NA & $\begin{array}{l}23 / 204 \\
(11 \%)\end{array}$ & $8 / 103(8 \%)$ \\
\hline \multicolumn{5}{|l|}{ Week 96} \\
\hline HBV DNA<29 IU/mL & $88(91 \%)$ & $57(97 \%)$ & $161(78 \%)$ & $86(80 \%)$ \\
\hline $\begin{array}{l}\text { ALT normalisation (AASLD } \\
\text { criteria) }^{a}\end{array}$ & $\begin{array}{l}46 / 94 \\
(49 \%)\end{array}$ & $\begin{array}{l}24 / 59 \\
(41 \%)\end{array}$ & $\begin{array}{l}119 / 207 \\
(57 \%)\end{array}$ & $\begin{array}{l}46 / 108 \\
(43 \%)\end{array}$ \\
\hline HBeAg loss & $N A^{b}$ & NA & $\begin{array}{l}55 / 204 \\
(27 \%)\end{array}$ & $\begin{array}{l}17 / 103 \\
(17 \%)\end{array}$ \\
\hline HBeAg seroconversion & NA & NA & $\begin{array}{l}47 / 204 \\
(23 \%)\end{array}$ & $\begin{array}{l}13 / 103 \\
(13 \%)\end{array}$ \\
\hline
\end{tabular}

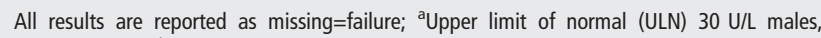
19 U/L females; ${ }^{b} \mathrm{NA}$, not applicable.

\section{IDDF2018-ABS-0113 THE SAFETY AND TOLERABILITY OF SOFI VEL/VOX FOR 8 OR 12 WEEKS IN $>1,000$ PATIENTS TREATED IN THE POLARIS-1, POLARIS-2, POLARIS-3, AND POLARIS-4 STUDIES: AN INTEGRATED ANALYSIS} ${ }^{6}$ Marc Bourliere, ${ }^{7}$ Tarik Asselah, ${ }^{8}$ Laurent Alric, ${ }^{9}$ Robert H Hyland, ${ }^{9}$ Luisa M Stamm, ${ }^{9} \mathrm{KC}$ Huang, ${ }^{9}$ Diana M Brainard, ${ }^{9}$ Chrstina SM Yip, Hai Cheng Huang ${ }^{9},{ }^{10}$ Mandana Khalili, ${ }^{11}$ Graham R Foster, ${ }^{12}$ Stuart C Gordon, ${ }^{13}$ Reddy K Rajender, ${ }^{14}$ Stefan Zeuzem, ${ }^{15}$ Ira M Jacobson, ${ }^{16}$ Curtis L Cooper, ${ }^{17}$ Alex J Thompson, ${ }^{18}$ Kris Kowdley, ${ }^{19}$ Eric Lawitz. ${ }^{1}$ Department of Gastroenterology, Hepatology and Endocrinology, Hannover Medical School, Hannove, Germany; ${ }^{2}$ Auckland Clinical Studies, Aucklan,, New Zealand; ${ }^{3}$ Centre Hospitalier de I'Universite de Montreal, Montreal, Canada; ${ }^{4}$ Alfred Hospital, Melbourne, Australia; ${ }^{5}$ Northwestern University, Chicago, USA; ${ }^{6}$ Hospital Saint Joseph, Marseille, France; ${ }^{7}$ Service d'Hepatologie, Hopital Beaujon, Universite Paris Diderot, Clichy, France; ${ }^{8}$ Internal MedecineDigestive Department, CHU Purpan, URM 152, Toulouse 3 University, Toulouse, France; ${ }^{9}$ Gilead Sciences, Inc., Foster City, CA, USA; ${ }^{10}$ University of California San Franciso, San Francisco, USA; ${ }^{11}$ Royal London Hospital, London, UK; ${ }^{12}$ Henry Ford Health System, Detroit, USA; ${ }^{13}$ University of Pennsylvania, Philadelphi, USA; ${ }^{14}$ Johann Wolfgang Goethe University Medical Center, Frankfurt, Germany; ${ }^{15}$ Department of Medicine, Mount Sinai Beth Israel, New York, USA; ${ }^{16}$ Ottawa Hospital Research Institute, Ottawa, Canada; ${ }^{17}$ Department of Gastroenterology, St. Vincent's Hospital, Melbourne, Australia; ${ }^{18}$ Swedish Medical Center, Seattle, USA; ${ }^{19}$ Texas Liver Institute, University of Texas Health Science Center, San Antonio, USA

\subsection{6/gutjnl-2018-IDDFbestabstracts.20}

Background The once-daily fixed-dose combination tablet of sofosbuvir/velpatasvir/voxilaprevir (SOF/VEL/VOX) was evaluated for the treatment of genotype 1-6 HCV infection in four phase 3 studies in patients with and without compensated cirrhosis. This analysis describes the safety of SOF/VEL/VOX across 4 Phase 3 studies.

Methods Treatment-emergent adverse events (AEs) and laboratory abnormalities were assessed in patients who received SOF/VEL/VOX or placebo for 12 weeks (POLARIS-1), SOF/ VEL/VOX or SOF/VEL for 12 weeks (POLARIS-4), or SOF/ 